\title{
SOBRE LAS ESTRATEGIAS METODOLÓGICAS DE LOS/AS EDUCADORES/AS PARA CONTRIBUIR AL EMPODERAMIENTO JUVENIL
}

\section{(ON EDUCATORS' METHODOLOGICAL STRATEGIES TO CONTRIBUTE TO YOUTH EMPOWERMENT)}

Carme Trull-Oliva

Universitat de Girona

Ángela Janer Hidalgo

Universidad Internacional de La Rioja

Laura Corbella Molina

Universitat Autònoma de Barcelona

Pere Soler-Masó

Juan González-Martínez

Universitat de Girona

DOI: $10.5944 / e d u c X X 1.30014$

Cómo referenciar este artículo/How to reference this article:

Trull-Oliva, C., Janer Hidalgo, Á., Corbella Molina, L., Soler-Masó, P. y González-Martínez, J. (2022). Sobre las estrategias metodológicas de los/as educadores/as para contribuir al empoderamiento juvenil. Educación XX1, 25(1), 459-483. https://doi.org/10.5944/educXX1.30014

Trull-Oliva, C., Janer Hidalgo, Á., Corbella Molina, L., Soler-Masó, P., \& González-Martínez, J. (2022). On educators' methodological strategies to contribute to youth empowerment. Educación XX1, 25(1), 459-483. https://doi.org/10.5944/educXX1.30014

\section{RESUMEN}

Si partimos de la idea de que los/as educadores/as son agentes importantes en el proceso de empoderamiento juvenil, a través de este estudio, se pretende analizar la visión de los profesionales de la educación sobre el uso de determinadas metodologías y estrategias, útiles y eficaces en los procesos empoderadores de los jóvenes. Se plantea un análisis cuantitativo por medio de un cuestionario autoadministrado, respondido por una muestra validada de 696 educadores de cinco comunidades del territorio español. Este análisis permite describir y analizar las estrategias metodológicas de los educadores y educadoras en su contribución a los procesos de empoderamiento juvenil y, determinar qué relación tiene el uso de esas estrategias con factores personales como el tamaño del territorio 
donde trabajan, la experiencia profesional, la formación específica en juventud o el ámbito de intervención en el que se ubican. En general, todos los profesionales están de acuerdo con la importancia de utilizar metodologías activas y participativas para promover y empoderar a los jóvenes, y declaran aplicarlas de manera generalizada junto a un acompañamiento del joven durante el proceso educativo. Más allá de este diagnóstico inicial, la investigación permite identificar diferencias importantes entre la aplicación de estas metodologías en el contexto rural y el urbano (donde se aplican más intensamente) o en función de si el/la educador/a ha obtenido formación específica en juventud; sin embargo, otras variables personales como la experiencia profesional o la edad del/de la educador/a no se muestran tan explicativas en este análisis. Finalmente, los ámbitos de ejercicio profesional nos permiten asociar determinados perfiles metodológicos.

\section{PALABRAS CLAVE}

Juventud, metodologías, empoderamiento juvenil, educadores

\section{ABSTRACT}

If we start from the idea that educators are important agents in the youth empowerment process, this study aims to analyse the vision of education professionals on the use of certain methodologies and strategies which are useful and effective in the processes of youth empowerment. A quantitative analysis is carried out by means of a self-administered questionnaire, answered by a validated sample of 696 educators from five communities in Spain. This analysis makes it possible to describe and analyse educators' methodological strategies in their contribution to youth empowerment processes and to determine how the use of these strategies relates to personal factors such as the size of the territory where they work, professional experience, specific youth training or the area of intervention in which they are located. All of the professionals agree on the importance of using active and participatory methodologies to promote and empower young people and declare that they are applied in a generalised way along with accompanying young people during the educational process. After this initial overview, the research allows us to identify important differences between the application of these methodologies in the rural and urban context (where they are applied more intensively) or depending on whether the educator has obtained specific training in youth; however, other personal variables such as professional experience or the age of the educator are not so explanatory in this analysis. Finally, the fields of professional practice allow us to associate certain methodological profiles. 


\section{KEY WORDS}

Youth, methodologies, youth empowerment, educators

\section{INTRODUCCIÓN}

El concepto empoderamiento sigue resultando aún hoy complejo, con cierta ambigüedad e imprecisión. Sin embargo, su uso se ha extendido y ha hecho fortuna en el ámbito socioeducativo, mayoritariamente referido a población adulta, si bien en las últimas décadas también entre los proyectos y programas juveniles, especialmente, entre aquellos colectivos en situaciones de vulnerabilidad y riesgo social.

Según Rappaport (1984), implica tener control de la propia vida. Ello nos obliga a pensar en términos de bienestar, competencia y fortaleza (Zimmerman, 2000). Por tanto, liga con la capacidad de elegir y de actuar teniendo en cuenta el conjunto de alternativas disponibles y los escenarios sociales en los que se materializan esas opciones (Bauman, 2010). Según San Pedro (2006), el empoderamiento puede entenderse como un ciclo de reflexión y acción que ayuda a tomar conciencia sobre las decisiones personales y, con ello, a transformar el propio rol en la sociedad. Por su parte, Peterson (2014) añade a la reflexión las consideraciones individual y comunitaria, y entiende que el empoderamiento promueve la capacidad para afrontar las dificultades individual y comunitariamente, con una implicación activa en la búsqueda de las posibles soluciones. El empoderamiento, cuando es potenciado por la educación, resulta estratégico en las luchas contra la recesión de los derechos subjetivos o de ciudadanía (Gonçalves y García, 2016).

En este marco conceptual, esta aportación parte del análisis documental (Úcar et al., 2016) y del modelo pedagógico capaz de orientar las intervenciones socioeducativas con jóvenes (Soler et al., 2017), ambos dentro del Proyecto HEBE ${ }^{1}$. Y, más en concreto, se interesa por el relato, la mirada y la capacidad analítica de los/as educadores/as, para profundizar en las estrategias de intervención educativa que, según ellos/as mismos/ as, favorecen el empoderamiento juvenil (EJ). Ofrecemos, pues, una mirada específica hacia la aplicación por parte de los/as profesionales de determinadas metodologías y estrategias, útiles y eficaces en los procesos empoderadores. 


\section{SOBRE LA FIGURA DEL EDUCADOR EN LOS PROCESOS DE EMPODERAMIENTO}

Parece reconocido que los educadores son claros agentes de empoderamiento (Rojas, 2014). En este sentido, Deutsch et al., (2020) sugieren la importancia de acompañar durante el proceso de aprendizaje y regular la presencia del educador en función de las necesidades educativas, mediante relaciones de confianza, respeto e interés. En esta línea, Ruff y Harrison (2020) proponen que los servicios juveniles podrían ofrecer más apoyo durante los períodos de transición, ya que es cuando los jóvenes experimentan el deseo de autonomía. Se trata de identificar con éxito las áreas donde la juventud necesita más ayuda para preparase y desarrollarse ante la vida, para poder fortalecer las aspiraciones y motivaciones personales (Young et al., 2020).

Hay diversidad de estudios (Kirk et al., 2015; Sullivan y Simonson, 2016; Vázquez-Fernández y Barrera-Algarín, 2016; Sung et al., 2017; Paños, 2017; Greene et al., 2018) que afirman que es necesario utilizar metodologías activas, centradas en los jóvenes, participativas y colaborativas para promover el empoderamiento de los jóvenes, ya que las propuestas pasivas, unidireccionales y centradas en el educador no son tan eficaces. Se recomiendan múltiples situaciones contextualizadas, reales y variadas (Paños, 2017; Ruff y Harrison, 2020).

Las aportaciones actuales también insisten en la importancia de fomentar roles equitativos y relaciones positivas entre los educadores y los jóvenes, el sentido de comunidad y el desarrollo de expectativas positivas. En este sentido, se recomienda trabajar en entornos que produzcan seguridad y apoyo a los jóvenes, creer en su éxito y sus potencialidades y promover la toma de decisiones de manera compartida, consciente y responsable. El educador debe facilitar que los jóvenes descubran sus propias capacidades para crear un cambio significativo (Greene et al., 2018). En definitiva, se insiste en la importancia de ofrecer a los jóvenes la oportunidad de autorregular su proceso educativo. Para ello, es clave crear escenarios para que tomen sus propias decisiones, asuman las responsabilidades derivadas y reflexionen sobre los resultados de sus acciones (Paños, 2017). Según Goyette et al. (2016), es fundamental involucrar a los/as jóvenes en las fases del diseño de cualquier programa de intervención.

Por otro lado, hay estudios que recomiendan adaptar y combinar la presencia del educador con el uso de las tecnologías de la información y la comunicación (TIC). Según algunos autores, integrar internet y las redes sociales en las acciones educativas favorece la creación de redes, la conexión espontánea e inmediata con servicios o agentes juveniles, y el acceso a un 
mundo de información útil y vital para ser y estar en el mundo (Sung et al., 2017; Greene et al., 2018; Greeson et al., 2020; Hongyee, 2020). Collura et al. (2019), además, recomiendan utilizar la comunidad como un recurso educativo, pues al participar en ella, los jóvenes se comprometen a delimitar problemáticas sociales, a identificar sus causas y a aplicar estrategias innovadoras para abordarlas. De esta manera, afrontan, de manera colaborativa, responsable y crítica, situaciones que afectan a la sociedad. Insisten también en la importancia de ayudar a los jóvenes a comprender los contextos sociopolíticos y de participación comunitaria. Greene et al. (2018) puntualizan que los espacios comunitarios deben pensarse como espacios de enseñanza, espacios inclusivos y en este mismo sentido Ruff y Harrison (2020) recomiendan promover la participación activa de los jóvenes y fortalecer el sentido de pertenencia a la comunidad a través del compromiso. Finalmente, queda claro que el contexto y la comunidad son fundamentales a la hora de promover el empoderamiento de los jóvenes. Conseguir que las personas se sientan parte de él, es clave para trabajar la capacidad de resiliencia de los jóvenes (Belykh, 2018; Prowell, 2019; Davidson y Carlin, 2019). Y no solo para desarrollar la capacidad de adaptación a situaciones diversas, sino también para educar en la gestión de las propias emociones y así, reforzar el bienestar y la transformación personal (Sullivan y Simonson, 2016; Belykh, 2018).

Desde la perspectiva institucional, el Council of Europe (2015) promueve el impulso de The Council of Europe Youth Work Portfolio. El Portfolio representa una propuesta de las competencias que los trabajadores juveniles deben tener para ejercer el trabajo juvenil. Entre sus 31 competencias ordenadas y clasificadas en 8 funciones, la función 3 implica apoyar y empoderar a los jóvenes para que den sentido a la sociedad en la que viven y se comprometan con ella. Esta función se concreta a partir de competencias como ayudar a los jóvenes a identificar y asumir responsabilidades, apoyar acciones individuales y colectivas para el cambio social, y favorecer el pensamiento crítico, la competencia y la confianza de los jóvenes. Finamente, en cuanto a las habilidades vinculadas a estas competencias, se destacan el pensamiento crítico, la escucha activa y la alfabetización política (Wagaman, 2016), y añade la conciencia crítica y la participación en la comunidad como predictores significativos del empoderamiento.

A partir de estas consideraciones, es pertinente, pues, preguntarnos en qué medida, los educadores/as que trabajan con jóvenes priorizan y aplican estas metodologías de acción educativa para promover el EJ. ¿Qué estrategias utilizan más para empoderar a los jóvenes? ¿Por qué? ¿Existen variables que influyan en su manera de contribuir al EJ? 
Conocer estas estrategias metodológicas en la intervención con jóvenes puede darnos muchas pistas acerca de cómo se favorece el empoderamiento y qué dificultades se encuentran los educadores a la hora de promoverlo.

\section{MATERIALES Y MÉTODOS}

Como avanzábamos, para dar respuesta a estas preguntas, y como complemento a otras aproximaciones metodológicas que exploraremos en el futuro, planteamos un análisis cuantitativo de la visión de los profesionales de la educación, por medio de la técnica de la encuesta, que nos permita un primer análisis que dé respuesta a los siguientes objetivos:

- Describir cuáles son las estrategias metodológicas que los educadores y educadoras utilizan más en su contribución a los procesos de empoderamiento juvenil.

- Determinar qué relación tiene el uso de esas estrategias metodológicas con factores personales como el tamaño del territorio donde trabajan los educadores y educadoras, la experiencia profesional, la formación específica en juventud o el ámbito de intervención en el que se ubican.

- Caracterizar los perfiles metodológicos de los/as educadores/ as en relación con las metodologías que usan en los procesos de empoderamiento juvenil, con especial atención a sus ámbitos de intervención.

Para ello, se ha aplicado un cuestionario ad hoc, diseñado y validado dentro del contexto de la investigación, con la siguiente estructura, precedida del correspondiente consentimiento informado: a) datos de perfil (edad, género, formación previa, ámbito de intervención, municipio), b) aplicación de estrategias metodológicas ligadas al EJ (variables escalares del grado de aplicación por parte del/de la educador/a cada una de las metodologías que, en la revisión de la literatura, resultan efectivas en procesos empoderadores), c) análisis de elementos contextuales institucionales y comunitarios que condicionan el trabajo del educador en relación con el EJ (variables escalares de presencia e impacto de los elementos contextuales que inciden en la acción de los/as educadores/as), d) contribución de la tarea del/de la educador/a a las nueve dimensiones del EJ (en escala de Likert), e) valoración de incidentes críticos y espacios en relación con el EJ (en escala de Likert). En este artículo focalizamos en analizar los bloques A y B únicamente. 
En relación con las metodologías analizadas en la sección B, en la Tabla 1 se recogen las que manan de la revisión teórica previa, con los códigos sintéticos usados en el análisis.

\section{Tabla 1}

Metodologías y estrategias analizadas

\begin{tabular}{ll}
\hline \multicolumn{1}{c}{ Metodología/estrategia } & \multicolumn{1}{c}{ Código } \\
\hline Acompañarlas durante el proceso de aprendizaje & Acompañamiento \\
\hline Educarlas en la gestión de las emociones & Emociones \\
\hline Ayudarlas a construir una imagen personal positiva & Imagen \\
\hline $\begin{array}{l}\text { Crear escenarios para que tomen sus propias decisiones y } \\
\text { asuman las responsabilidades derivadas }\end{array}$ & Decisiones \\
\hline $\begin{array}{l}\text { Desarrollar de una manera flexible las acciones educati- } \\
\text { vas previstas }\end{array}$ & Flexibilidad \\
\hline $\begin{array}{l}\text { Adaptar las acciones educativas a sus características per- } \\
\text { sonales }\end{array}$ & Adaptación \\
\hline Utilizar metodologías activas y participativas & Activas \\
\hline $\begin{array}{l}\text { Integrar internet y las redes sociales en las acciones edu- } \\
\text { cativas }\end{array}$ & Internet \\
\hline $\begin{array}{l}\text { Desarrollar el proceso educativo conjuntamente con las } \\
\text { personas jóvenes }\end{array}$ & Codiseño \\
\hline Gestionar las situaciones difíciles & Dificultades \\
\hline Trabajar la resiliencia & Resiliencia \\
\hline $\begin{array}{l}\text { Potenciar el desarrollo de las capacidades comunicativas } \\
\text { y de negociación }\end{array}$ & Comunicación \\
\hline $\begin{array}{l}\text { Dar espacios para que reflexionen sobre los resultados de } \\
\text { sus acciones }\end{array}$ & Reflexión \\
\hline Fomentar el trabajo colaborativo & Trabajo colaborativo \\
\hline Utilizar la comunidad como un recurso educativo & Comunidad \\
\hline $\begin{array}{l}\text { Promover la reflexión acerca del propio proceso de } \\
\text { aprendizaje }\end{array}$ & Metacognición \\
\hline $\begin{array}{l}\text { Regular la presencia del educador en función de las nece- } \\
\text { sidades educativas }\end{array}$ & Presencia \\
\hline & \\
\hline
\end{tabular}

El cuestionario fue diseñado y validado entre enero de 2018 y septiembre de 2019, en un proceso que, por economía del espacio, ahora solo resumimos en las siguientes fases: selección del acervo documental (revisión de la literatura y de los documentos de investigación previos para determinar el foco del instrumento), aislamiento de las variables relevantes 
(y análisis de las posibilidades de sondeo), definición de la estructura del cuestionario, redacción y revisión de los reactivos (validación por 17 expertos de diferentes perfiles de los ámbitos socioeducativo y universitario), primer pilotaje, segunda revisión de los ítems a partir del pilotaje, digitalización del instrumento dentro del servidor institucional de formularios, pilotaje del desarrollo (tras la digitalización) y entrevistas cognitivas de cierre (a 6 potenciales informantes).

Se administró on-line durante el último trimestre de 2019, a una muestra accesible de profesionales de la educación de los cuatro ámbitos de intervención que a priori habíamos definido (educación especializada, formación ocupacional, animación sociocultural y ocio, educación formal) (Froufe, 1997), y en los cinco territorios que el proyecto había definido también a priori (Catalunya, Euskadi, Galicia, Madrid y Andalucía), a los que se contactó por medio de mailing abierto (profesorado) o cerrado (profesionales socioeducativos), en tres sucesivas llamadas.

Se consiguió la respuesta depurada de 696 sujetos, con los siguientes datos de perfil: un $66 \%$ eran mujeres y un $32 \%$ fueron hombres (un $1 \%$ se categorizó como género no binario y el restante $1 \%$ prefirió no identificar su género); un $53 \%$ procede de Catalunya y un $23 \%$ de Euskadi, territorios sobrerrepresentados en la muestra (12\% Andalucía, 5\% Galicia, 6\% Madrid). Según el tamaño de la localidad donde ejercen profesionalmente, predominan los entornos rurales o núcleos de población pequeños, por debajo de los 20000 habitantes (32\%), seguidos de las grandes ciudades entre los 100001 y los 500000 habitantes (23\%); el resto se distribuye entre municipios medianos (20001-50000, 21\%; 50001-100000, 11\%) y las grandes conurbaciones (más de 500001 habitantes, 13\%). La gran mayoría de la población declara una experiencia considerable (superior a 11 años, 67\%, entre 6 y 10 años, 14\%), mientras que es minoritaria la proporción de profesionales por debajo de los cinco años de experiencia profesional (19\%). En cuanto al ámbito de intervención, un 59\% ejerce en el ámbito formal, un $7 \%$ en el ámbito ocupacional, un $22 \%$ en la animación sociocultural y un $12 \%$ en la educación especializada.

\section{RESULTADOS}

En este apartado se presentan, en primer lugar, los resultados sobre cuáles son las metodologías que los/as educadores/as utilizan en los procesos de acompañamiento al EJ. Y, en segundo lugar, y por economía del espacio, únicamente las diferencias según experiencia laboral, formación previa o el ámbito de intervención en el que trabajan, que son las que más explicativas se han mostrado en este punto. 


\section{Uso de metodologías orientadas al EJ}

Como se aprecia en la Tabla 2, en escala de 1 a 4, los/as educadores/as indican que utilizan todas las metodologías consideradas empoderadoras. Aunque las diferencias son mínimas, las más utilizadas (3.4 sobre 4) son el acompañamiento en el proceso de aprendizaje y el uso de metodologías activas y participativas. Por el contrario, las menos utilizadas (2.9 sobre 4), aunque con una puntuación que indica una utilización alta, son el trabajo de la resiliencia, el uso de la comunidad como recurso educativo, la regulación de la presencia del educador y el codiseño del proceso educativo junto con las personas jóvenes.

\section{Tabla 2}

Uso de metodologías orientadas al desarrollo del EJ

\begin{tabular}{lcc}
\hline & MEDIA & SD \\
\hline Acompañamiento & 3.4 & .657 \\
\hline Activas & 3.4 & .758 \\
\hline Decisiones & 3.3 & .738 \\
\hline Dificultades & 3.3 & .668 \\
\hline Trabajo colaborativo & 3.2 & .694 \\
\hline Adaptación & 3.2 & .729 \\
\hline Flexibilidad & 3.2 & .631 \\
\hline Imagen & 3.2 & .814 \\
\hline Comunicación & 3.1 & .758 \\
\hline Reflexión & 3.1 & .701 \\
\hline Metacognición & 3.1 & .812 \\
\hline Emociones & 3.0 & .751 \\
\hline Internet & 3.0 & .739 \\
\hline Resiliencia & 2.9 & .727 \\
\hline Comunidad & 2.9 & .833 \\
\hline Presencia & 2.9 & .734 \\
\hline Codiseño & 2.9 & .771 \\
\hline
\end{tabular}

En líneas generales, se observa que las metodologías activas y participativas son de las más trabajadas mientras que la implicación del joven en el diseño del proceso de aprendizaje y el trabajo con la comunidad están valoradas en las últimas posiciones. 


\section{INCIDENCIA DE LAS VARIABLES DE PERFIL EN EL USO DE LAS METODOLOGÍAS ORIENTADAS AL EJ}

En relación con el tamaño del municipio donde trabaja el/la educador/a, encontramos la siguiente información (Tabla 3):

Tabla 3

Incidencia de la variable tamaño de la localidad

\begin{tabular}{|c|c|c|c|c|c|c|c|c|c|c|c|}
\hline & \multicolumn{2}{|c|}{$<20000$} & \multicolumn{2}{|c|}{$\begin{array}{l}20001- \\
50000\end{array}$} & \multicolumn{2}{|c|}{$\begin{array}{l}50001- \\
100000\end{array}$} & \multicolumn{2}{|c|}{$\begin{array}{l}100001- \\
500000\end{array}$} & \multicolumn{2}{|c|}{$>500001$} & \multirow{2}{*}{ Sig. } \\
\hline & $\mathbf{M}$ & SD & M & SD & $\mathbf{M}$ & SD & M & SD & M & SD & \\
\hline Acompañamiento & 3.3 & .669 & 3.3 & .626 & 3.5 & .661 & 3.4 & .668 & 3.5 & .636 & .088 \\
\hline Emociones & 3.0 & .742 & 2.9 & .763 & 3.1 & .774 & 3.0 & .756 & 3.3 & .731 & $.004 *$ \\
\hline Imagen & 3.1 & .685 & 3.0 & .789 & 3.2 & .784 & 3.2 & .746 & 3.3 & .699 & $.034 *$ \\
\hline Decisiones & 3.3 & .628 & 3.3 & .714 & 3.2 & .695 & 3.3 & .671 & 3.4 & .660 & .474 \\
\hline Flexibilidad & 3.2 & .688 & 3.1 & .708 & 3.1 & .757 & 3.2 & .690 & 3.3 & .632 & .282 \\
\hline Adaptación & 3.2 & .678 & 3.0 & .763 & 3.1 & .848 & 3.3 & .689 & 3.2 & .728 & $.044 *$ \\
\hline Activas & 3.4 & .605 & 3.4 & .651 & 3.4 & .695 & 3.4 & .619 & 3.5 & .634 & .708 \\
\hline Internet & 2.9 & .783 & 3.0 & .782 & 3.0 & .887 & 3.0 & .828 & 3.0 & .853 & .700 \\
\hline Codiseño & 2.9 & .713 & 2.8 & .790 & 2.9 & .821 & 2.9 & .774 & 3.0 & .730 & .522 \\
\hline Dificultades & 3.2 & .642 & 3.2 & .761 & 3.2 & .767 & 3.3 & .711 & 3.5 & .634 & $.017 *$ \\
\hline Resiliencia & 2.9 & .727 & 2.7 & .926 & 2.9 & .833 & 3.0 & .812 & 3.2 & .722 & $.001 \%$ \\
\hline Comunicación & 3.1 & .686 & 3.0 & .820 & 3.1 & .774 & 3.2 & .740 & 3.3 & .757 & $.021 *$ \\
\hline Reflexión & 3.1 & .685 & 3.1 & .720 & 3.0 & .769 & 3.1 & .761 & 3.2 & .829 & .629 \\
\hline Trabajo colaborativo & 3.1 & .740 & 3.2 & .690 & 3.2 & .772 & 3.3 & .686 & 3.3 & .768 & .134 \\
\hline Comunidad & 2.9 & .780 & 2.9 & .839 & 2.9 & .876 & 2.8 & .917 & 3.0 & .758 & .496 \\
\hline Metacognición & 3.1 & .734 & 3.0 & .824 & 3.1 & .692 & 3.2 & .678 & 3.3 & .678 & $.011 \%$ \\
\hline Presencia & 2.9 & .712 & 2.8 & .778 & 2.7 & .880 & 3.0 & .760 & 3.0 & .801 & .079 \\
\hline
\end{tabular}

* diferencias significativas en la variable según los test ANOVA

Existen diferencias significativas en 7 de las 17 metodologías. En el uso de metodologías en función de esta variable, en todo caso, se observa un mismo patrón, que las metodologías que más pueden contribuir al EJ se usan más en el contexto urbano que en el rural, con independencia de que en unos casos esas diferencias sean significativas o no. Lo cual, hasta cierto punto, puede explicarse por las limitaciones de la muestra, de tal modo que sea plausible pensar que en una muestra mayor todas esas diferencias lo fueran. Siguiendo el patrón, las medias de puntuación más bajas se sitúan en las poblaciones de menos de 100000 habitantes mientras que las medias más altas 
están ubicadas en poblaciones de más de 500000 habitantes, compartiendo la misma valoración que las poblaciones de 100000 a 500000 en tres metodologías (internet, trabajo colaborativo y presencia). Con dos excepciones: (1) el uso de la adaptación del proceso de aprendizaje a la persona tiene la media más alta en poblaciones grandes; y (2) el acompañamiento tiene la media más alta tanto en poblaciones grandes como en poblaciones medianas.

En relación con la experiencia profesional del/de la educador/a, encontramos la siguiente información (tabla 4):

Tabla 4

Incidencia de la experiencia profesional

\begin{tabular}{|c|c|c|c|c|c|c|c|c|c|}
\hline & \multicolumn{2}{|c|}{$<1$ año } & \multicolumn{2}{|c|}{ 1-5 años } & \multicolumn{2}{|c|}{ 6-10 años } & \multicolumn{2}{|c|}{$>11$ años } & \multirow{2}{*}{ Sig. } \\
\hline & $\mathbf{M}$ & SD & $\mathbf{M}$ & SD & $\mathbf{M}$ & SD & M & SD & \\
\hline Acompañamiento & 3.3 & .602 & 3.2 & .691 & 3.4 & .709 & 3.4 & .632 & $.008^{*}$ \\
\hline Emociones & 3.3 & .683 & 2.9 & .810 & 3.0 & .753 & 3.1 & .746 & .244 \\
\hline Imagen & 2.9 & .680 & 3.1 & .769 & 3.3 & .737 & 3.2 & .730 & .111 \\
\hline Decisiones & 3.4 & .719 & 3.2 & .684 & 3.3 & .651 & 3.3 & .666 & .422 \\
\hline Flexibilidad & 3.4 & .619 & 3.1 & .745 & 3.1 & .745 & 3.2 & .670 & .148 \\
\hline Adaptación & 3.4 & .512 & 3.1 & .759 & 3.2 & .756 & 3.2 & .721 & .275 \\
\hline Activas & 3.7 & .479 & 3.3 & .656 & 3.4 & .640 & 3.4 & .625 & .094 \\
\hline Internet & 2.8 & .750 & 2.9 & .838 & 3.0 & .766 & 3.0 & .820 & .656 \\
\hline Codiseño & 3.1 & .680 & 2.9 & .727 & 3.0 & .709 & 2.9 & .778 & .403 \\
\hline Dificultades & 3.2 & .655 & 3.1 & .747 & 3.3 & .647 & 3.3 & .701 & .200 \\
\hline Resiliencia & 2.9 & .680 & 2.9 & .888 & 2.9 & .797 & 3.0 & .800 & .861 \\
\hline Comunicación & 3.1 & .772 & 3.1 & .811 & 3.1 & .768 & 3.2 & .731 & .522 \\
\hline Reflexión & 3.1 & .719 & 3.1 & .731 & 3.1 & .739 & 3.1 & .744 & .904 \\
\hline Trabajo colaborativo & 3.3 & .602 & 3.2 & .700 & 3.1 & .741 & 3.2 & .735 & .633 \\
\hline Comunidad & 3.1 & .719 & 2.8 & .873 & 2.9 & .795 & 2.9 & .835 & .567 \\
\hline Metacognición & 2.9 & .772 & 3.1 & .735 & 3.1 & .751 & 3.1 & .730 & .572 \\
\hline Presencia & 2.9 & .619 & 2.9 & .713 & 2.9 & .745 & 2.9 & .797 & .992 \\
\hline
\end{tabular}

* diferencias significativas en la variable según los test ANOVA

No existen diferencias significativas en el uso de las diferentes metodologías según los años de experiencia profesional, exceptuando el uso del acompañamiento que obtiene las medias más altas a partir de los 5 años de experiencia. Aunque las medias más altas y más bajas están repartidas, y teniendo en cuenta que no hay un grado de significación en ninguna de ellas, observamos como hay ciertas metodologías que se usan más a mayor 
experiencia y otras que se usan más a menor experiencia. Las metodologías enfocadas en trabajar capacidades personales (resiliencia, comunicación, metacognición, dificultades) son utilizadas por educadores con un mayor grado de experiencia mientras que aquellas metodologías centradas en aspectos colectivos, participativos y comunitarios son utilizadas en mayor medida por los educadores con menos experiencia (p. ej. activas, codiseño, trabajo colaborativo, comunidad, flexibilidad, adaptación).

En cuanto a la incidencia de la variable de formación específica en juventud, con la información de que disponíamos sobre la formación específica recibida, hemos aislado diferentes grupos (ninguna formación, formación inicial, formación continua, formación de posgrado, dos formaciones o todas las formaciones). A partir de su análisis se apuntan diferencias significativas en 10 de las 17 , recogidas en la tabla 5.

Tabla 5

Incidencia de la variable formación específica en juventud

\begin{tabular}{lccccccc}
\hline & $\begin{array}{c}\text { Solo } \\
\text { FI }\end{array}$ & $\begin{array}{c}\text { Solo } \\
\text { FC }\end{array}$ & $\begin{array}{c}\text { Solo } \\
\text { FP }\end{array}$ & Dos & Tres & Ning. & Sig. \\
\hline Acompañamiento & 3.33 & 3.41 & 3.48 & 3.38 & 3.61 & 3.37 & .449 \\
\hline Emociones & 3.00 & 3.14 & 3.02 & 3.16 & 3.29 & 2.90 & $.002^{*}$ \\
\hline Imagen & 3.13 & 3.30 & 3.12 & 3.24 & 3.50 & 3.01 & $.000^{*}$ \\
\hline Decisiones & 3.39 & 3.30 & 3.29 & 3.38 & 3.54 & 3.20 & $.032^{*}$ \\
\hline Flexibilidad & 3.04 & 3.20 & 3.33 & 3.22 & 3.36 & 3.10 & .088 \\
\hline Adaptación & 2.98 & 3.26 & 3.29 & 3.26 & 3.32 & 3.09 & $.016^{*}$ \\
\hline Activas & 3.33 & 3.47 & 3.43 & 3.45 & 3.43 & 3.30 & $.046^{*}$ \\
\hline Internet & 2.93 & 3.01 & 2.83 & 2.95 & 3.21 & 2.93 & .411 \\
\hline Codiseño & 2.91 & 2.94 & 2.90 & 2.93 & 2.96 & 2.80 & .403 \\
\hline Dificultades & 3.22 & 3.30 & 3.31 & 3.32 & 3.43 & 3.18 & .275 \\
\hline Resiliencia & 2.93 & 3.08 & 2.79 & 3.02 & 3.29 & 2.78 & $.000^{*}$ \\
\hline Comunicación & 3.22 & 3.22 & 3.14 & 3.25 & 3.25 & 3.01 & $.023^{*}$ \\
\hline Reflexión & 3.20 & 3.11 & 3.10 & 3.21 & 3.50 & 2.96 & $.001^{*}$ \\
\hline Trabajo colaborativo & 3.19 & 3.23 & 3.14 & 3.30 & 3.32 & 3.18 & .673 \\
\hline Comunidad & 2.98 & 2.99 & 3.00 & 3.07 & 3.07 & 2.76 & $.005^{*}$ \\
\hline Metacognición & 3.19 & 3.19 & 3.07 & 3.12 & 3.29 & 2.98 & $.027^{*}$ \\
\hline Presencia & 3.04 & 2.95 & 2.95 & 2.96 & 2.89 & 2.77 & .067 \\
\hline & & & & & & &
\end{tabular}

* diferencias significativas en la variable según los test ANOVA 
Aquellos/as educadores/as con formación en juventud tienen los valores más bajos en la mayoría de casos, exceptuando las metodologías enfocadas al acompañamiento, la flexibilidad, la adaptación a la persona. En cambio, los valores más altos se encuentran en los que han tenido todos los tipos de formación. Ahora bien, la diferencia entre no tener formación y tenerla en la FI o en la FC o en la FP no es significativa. Empieza a serlo, en determinadas metodologías, cuando acumulamos dos experiencias de formación; y mejora cuando los sujetos se forman específicamente en juventud en todas las posibles experiencias formativas analizadas.

Finalmente, en relación con el ámbito de intervención del/de la educador/a, encontramos la siguiente información (Tabla 6):

\section{Tabla 6}

Incidencia de la variable ámbito de intervención del educador

\begin{tabular}{|c|c|c|c|c|c|c|c|c|c|}
\hline & \multicolumn{2}{|c|}{ Ocup. } & \multicolumn{2}{|c|}{ Espec. } & \multicolumn{2}{|c|}{ Socioc. } & \multicolumn{2}{|c|}{ Formal } & \multirow{2}{*}{ Sig. } \\
\hline & $\mathbf{M}$ & SD & $\mathbf{M}$ & SD & M & SD & $\mathbf{M}$ & SD & \\
\hline Acompañamiento & 3.3 & .671 & 3.3 & .704 & 3.2 & .722 & 3.5 & .594 & $.000 *$ \\
\hline Emociones & 2.7 & .751 & 3.5 & .614 & 3.1 & .708 & 3.0 & .765 & $.000 *$ \\
\hline Imagen & 3.2 & .748 & 3.5 & .614 & 3.1 & .732 & 3.1 & .748 & $.000 *$ \\
\hline Decisiones & 3.4 & .663 & 3.5 & .593 & 3.4 & .593 & 3.2 & .691 & $.000 *$ \\
\hline Flexibilidad & 3.1 & .818 & 3.3 & .649 & 3.1 & .750 & 3.2 & .664 & .232 \\
\hline Adaptación & 3.4 & .639 & 3.5 & .688 & 3.1 & .822 & 3.1 & .695 & $.000 *$ \\
\hline Activas & 3.5 & .580 & 3.5 & .549 & 3.5 & .598 & 3.3 & .651 & $.000 *$ \\
\hline Internet & 3.2 & .708 & 2.7 & .791 & 3.0 & .761 & 3.0 & .833 & $.000 *$ \\
\hline Codiseño & 3.1 & .712 & 3.1 & .652 & 3.1 & .710 & 2.8 & .776 & $.000 *$ \\
\hline Dificultades & 3.1 & .783 & 3.6 & .565 & 3.2 & .713 & 3.2 & 695 & $.000 *$ \\
\hline Resiliencia & 3.0 & .869 & 3.4 & .667 & 2.9 & .803 & 2.9 & .800 & $.000 *$ \\
\hline Comunicación & 3.3 & .784 & 3.4 & 639 & 3.0 & .747 & 3.1 & .760 & $.006 *$ \\
\hline Reflexión & 3.1 & .857 & 3.4 & .601 & 3.2 & .657 & 3.0 & .758 & $.000 *$ \\
\hline Trabajo colaborativo & 3.3 & .701 & 3.1 & .760 & 3.4 & .646 & 3.2 & .740 & $.001 \%$ \\
\hline Comunidad & 3.0 & .845 & 3.2 & .823 & 3.1 & .791 & 2.8 & .822 & $.000 *$ \\
\hline Metacognición & 3.3 & .745 & 3.2 & .716 & 3.0 & .795 & 3.1 & .705 & $.007 *$ \\
\hline Presencia & 2.9 & .931 & 3.1 & .662 & 2.9 & .724 & 2.8 & .780 & $.020 *$ \\
\hline
\end{tabular}

* diferencias significativas en la variable según los test ANOVA

Todas las metodologías presentan diferencias significativas según esta variable, excepto la flexibilidad en la acción educativa. 
A simple vista, se puede observar la preponderancia del ámbito de especializada en el uso de metodologías para el empoderamiento (obtiene las medias más altas en 13 de las 17 metodologías), obteniendo las medias más bajas solo en el uso de internet y el trabajo colaborativo. Por el contrario, también se observa que el ámbito de la educación formal es el que obtiene las medias más bajas en el uso de metodologías para el empoderamiento (9 de las 17 metodologías) y solo destaca en el uso del acompañamiento al joven. El ámbito sociocultural también obtiene las medias más bajas en 7 metodologías. Sí destaca, siguiendo con las características del ámbito, en el uso de metodologías activas, codiseño de los aprendizajes (aunque está igual valorado en todo el ámbito social), y en el trabajo colaborativo. Por último, en el ámbito ocupacional, destacan las medias más altas en el uso de metodologías activas, el uso de internet, el codiseño de aprendizajes, y la metacognición.

A partir de esta primera descripción, profundizamos en el análisis de los ámbitos de acción socioeducativa por medio un análisis factorial y encontramos el siguiente modelo (tabla 7), con una KMO de 0.905 y significación de .000:

\section{Tabla 7}

Análisis factorial de las estrategias metodológicas

\begin{tabular}{lccc}
\hline \multicolumn{4}{c}{ Matriz de componentes rotados } \\
& \multicolumn{3}{c}{ Componente $^{\mathbf{2}}$} \\
\cline { 2 - 4 } & $\mathbf{1}$ & $\mathbf{2}$ & $\mathbf{3}$ \\
\hline Emociones & .794 & \\
\hline Imagen & .739 & .217 \\
\hline Resiliencia & .658 & .344 \\
\hline Reflexión & .620 & .368 & \\
\hline Comunicación & .619 & .278 & \\
\hline Dificultades & .597 & & .420 \\
\hline Metacognición & .478 & .405 & .248 \\
\hline Decisiones & .476 & .403 & \\
\hline Trabajo colaborativo & & .723 & .215 \\
\hline Met. Activas & & .670 & \\
\hline Comunidad & .280 & .639 & .230 \\
\hline Internet & .212 & .638 & .796 \\
\hline Codiseño & .259 & .597 & \\
\hline Flexibilidad & & .224 & \\
\hline
\end{tabular}




\begin{tabular}{|c|c|c|c|}
\hline Adaptación & .223 & & .674 \\
\hline Acompañamiento & .249 & & .560 \\
\hline Presencia & .318 & .217 & .431 \\
\hline
\end{tabular}

a. La rotación ha convergido en 6 iteraciones

Fuente. Elaboración propia

Estos tres perfiles corresponden con tres arquetipos de profesional. Analizamos estas tres comunalidades que sugieren tres perfiles metodológicos bajo una perspectiva de ámbitos tradicionales de la acción socioeducativa con jóvenes. En ese sentido, convertimos cada uno de los factores en variable regresiva y vemos qué diferencias se establecen en cada uno de los ámbitos profesionales analizados en el estudio (Tabla 8).

\section{Tabla 8}

Incidencia de los diferentes perfiles metodológicos en los ámbitos profesionales

\begin{tabular}{cccc}
\hline & Tipo A & Tipo B & Tipo C \\
\hline Ocupacional & -.099 & .352 & -.032 \\
\hline Especializada & .714 & -.102 & .041 \\
\hline Sociocultural & -.009 & .361 & -.322 \\
\hline Formal & -.126 & -.154 & .109 \\
\hline Sig. (ANOVA) & .000 & .000 & .000 \\
\hline
\end{tabular}

A partir de aquí, apreciamos una correspondencia entre esos perfiles metodológicos y los ámbitos de ejercicio profesional: el tipo A es más frecuente en la educación especializada, y el tipo $\mathrm{C}$ en la educación formal; el tipo B, más centrado en los elementos sociales y comunitarios, destaca tanto en la formación ocupacional como en la animación sociocultural.

Vinculando el análisis estadístico con la matriz de componentes y el análisis regresivo, se observa la preponderancia del ámbito de especializada en el uso de metodologías para el empoderamiento y asociada al perfil metodológico de tipo A. El ámbito de la educación formal es el que obtiene las medias más bajas en el uso de estas mismas metodologías y está asociado al perfil metodológico de tipo $\mathrm{C}$. El análisis llevado a cabo, pues, nos permite concluir la posible existencia de unos perfiles metodológicos que podrían explicar diferentes características compartidas entre los educadores en los distintos ámbitos de ejercicio profesional estudiados. 


\section{DISCUSIÓN Y CONCLUSIONES}

El estudio realizado nos confirma la presencia de todas las metodologías analizadas a la hora de abordar el empoderamiento de los jóvenes y, además, nos muestra diferencias significativas en cuanto a su elección y su aplicación cuando la finalidad es el EJ.

El acompañamiento del joven aparece como la estrategia metodológica más utilizada junto con las metodologías activas y participativas, en consonancia con la importancia y la necesidad que los estudios les atribuyen a la hora de promover y ser eficaces en el EJ (Kirk et al., 2015; Sullivan \& Simonson, 2016; Vázquez-Fernández y Barrera-Algarín, 2016; Sung et al., 2017; Paños, 2017; Greene et al., 2018).

Aunque todas las estrategias metodológicas parecen gozar de amplio predicamento, se constata que la implicación del joven en el diseño del proceso de aprendizaje y su trabajo con la comunidad están valoradas en las últimas posiciones en cuanto a su uso y aplicación. Habría que profundizar más en este aspecto, pero, con los datos obtenidos, una posible hipótesis nos llevaría al predominio de la intervención centrada sobre el joven a nivel individual o grupo concreto al margen del posible y necesario trabajo con la comunidad y con el contexto. Tenemos aún el reto pendiente en ese sentido. Hay que recordar que justamente la conciencia crítica y la participación en la comunidad se definen como predictores significativos del empoderamiento juvenil (Wagaman, 2016) y que el contexto y la comunidad se han identificado como fundamentales a la hora de promover el empoderamiento de los jóvenes (Belykh, 2018; Prowell, 2019; Davidson y Carlin, 2019). En este sentido, debemos aprovechar la comunidad como un recurso educativo. Los/as jóvenes deben delimitar problemáticas sociales concretas, identificar sus causas y saber aplicar estrategias al respecto. Para ellos/as, es necesaria cierta cesión de poder por parte de la comunidad ya que, respecto al poder, lo que verdaderamente es importante es aprender a compartirlo (Soler et al., 2017).

Nos gustaría hacer unos apuntes específicos a las variables de perfil analizadas. En primer lugar, el tamaño del territorio puede afectar al uso de las metodologías para el empoderamiento de los jóvenes, pero no es determinante. Existe una tendencia a usar determinadas metodologías según el tamaño del territorio, lo que nos hace pensar que las metodologías para el empoderamiento pueden variar según la comunidad y que, por tanto, una futura línea de estudio sería analizar las diferencias del trabajo socioeducativo para el empoderamiento juvenil en comunidades diversas. 
En segundo lugar, los datos recopilados y analizados nos permiten concluir que en la muestra de este estudio la experiencia profesional no es una variable que influya en el uso de metodologías para favorecer el EJ, pues parece que no hay una relación clara entre experiencia profesional y uso metodológico, y por eso la experiencia no aparece como una variable significativa. Sin embargo, debemos ser cautos, pues la muestra presenta limitaciones a este respecto; y, en concreto, los profesionales con menos de 5 años de experiencia están infrarrepresentados. Con todo, si se analiza la tendencia a pesar de las limitaciones, se observa que los educadores noveles utilizan metodologías más dinámicas, participativas y comunitarias, acaso amparados en el dinamismo y en la ilusión del comienzo de la trayectoria profesional. Por el contrario, los educadores más experimentados se centran en mayor medida en el uso de metodologías más individuales, enfocadas al desarrollo de capacidades personales y menos supeditadas a los condicionantes contextuales. Ello explicaría por qué en esta muestra con profesionales con larga trayectoria la dimensión comunitaria del empoderamiento, aun con una buena puntuación, tiene una de las puntuaciones más bajas. Habría posibles explicaciones ante esta constatación en el uso de metodologías: la necesidad por parte de los educadores noveles de buscar una mayor seguridad ante los trabajos colectivos (con la implicación de otros profesionales y servicios) o el cansancio que puede generar en los educadores más experimentados el mantener activa una red comunitaria habitualmente sin los recursos ni el reconocimiento requeridos, entre otras.

En cualquier caso, la dimensión comunitaria del EJ en esta muestra tiene recorrido por delante. En este sentido hay que pensar en más herramientas y recursos para facilitar y estimular a los educadores y profesionales con más experiencia ante este reto. La incidencia de un buen trabajo con la comunidad tiene efectos educativos y transformadores de gran amplitud (To et al., 2020; Collura et al., 2019).

En tercer lugar, la formación de los educadores es otro aspecto que merece atención a luz de los datos obtenidos y a la espera de otros estudios que aporten más investigación sobre este tema específico. Nuestros resultados obtenidos indican que la formación específica en juventud tiene incidencia tanto en la diversidad como en la intensidad de aplicación de metodologías para el EJ, únicamente cuando se realiza de manera reiterada. Resulta relevante cuando se trata de trayectorias que ponen en evidencia un claro interés en las temáticas juveniles y una voluntad manifiesta de formación específica en juventud desde la formación inicial, pasando por la formación continua e incluyendo la formación de postgrado. En conjunto, sí incide en la aplicación de estrategias metodológicas; pero incide menos cuando se ha dado únicamente en uno de estos ámbitos formativos. En esta investigación, 
un 39\% de los educadores no habían recibido formación específica en temas de juventud, y no muestran ninguna diferencia significativa en la aplicación de metodologías para el empoderamiento con el $43 \%$ de educadores que sí la han recibido en alguna etapa. En este sentido hay que cuestionarse qué tipo de formación específica en juventud han recibido estos profesionales en los distintos niveles citados y qué recursos y herramientas se proporcionan para la acción con los jóvenes.

Por último, el ámbito educativo es la variable más determinante en el uso de metodologías para el empoderamiento juvenil, según los resultados alcanzados. Las propias metodologías analizadas confirman y validan la existencia de determinados perfiles profesionales en la intervención socioeducativa, según las estrategias que en cada caso articulan. Gracias al análisis regresivo, encontramos tres perfiles metodológicos asociados a los ámbitos educativos analizados. El ámbito sociocultural destaca en el uso de metodologías activas, codiseño de los aprendizajes y en el trabajo colaborativo. Por último, el ámbito ocupacional obtiene las medias más altas en el uso de metodologías activas, el uso de internet, el codiseño de aprendizajes, y la metacoginición.

El tipo A, vinculado al ámbito de la educación especializada, se trata de un modelo más holístico y clásico, centrado en la relación y en las capacidades personales de la persona. Tiene en cuenta la comunidad. Clásico, porque no utiliza metodologías activas ni internet y es poco flexible. Centrado en la persona, porque utiliza poco el trabajo colaborativo. El tipo B, vinculado a los ámbitos de formación ocupacional y animación sociocultural, es un modelo centrado en la dimensión social, en la interacción y en la presencia en los espacios públicos (internet, comunidad, grupos). No profundiza en las capacidades personales e individuales (emoción, imagen, resiliencia, dificultades, etc.). No predominan ni la adaptación ni el acompañamiento. El tipo C, vinculado al ámbito de la educación formal, se centra en la dimensión individual, con poca atención a la relación afectiva y la interacción social. Quizás el arquetipo profesional menos empoderador, pues profundiza menos en las emociones, la reflexión, la comunicación, la comunidad, y en el trabajo colaborativo. Por el contrario, está muy centrado en las cuestiones más metodológicas desde una perspectiva clásica (codiseño, flexibilidad, adaptación, acompañamiento, metodologías activas, etc.) y no tanto en la persona y en sus interacciones.

Llegados a este punto, es justo situar los avances de la investigación, junto con sus limitaciones. Explorar la perspectiva de los/as profesionales de la educación por medio de un cuestionario cuantitativo, lo cual implica grandes posibilidades en cuanto a la extensión cubierta, también supone un reto en términos de intensión. En ese sentido, los resultados que hemos 
compartido en este artículo son especialmente relevantes por la novedad (hasta donde conocemos, no solo se trata de una aproximación al objeto de estudio novedosa en general, sino también desde el punto de vista metodológico). La muestra, sin duda, supone uno de los puntos críticos: por un lado, es una muestra amplia y diversa, en consonancia con la diversidad del colectivo al que representa; por otro, nace como muestra accesible y, por tanto, tensiona en muchos puntos el principio de equiprobabilidad. De todo ello se deriva una cierta cautela como premisa máxima al valorar los avances del conocimiento que presentamos: si bien en líneas generales los consideramos sólidos, las futuras investigaciones deberán ir confirmándolos con nuevas muestras (más amplias y representativas) y con el concurso de estrategias metodológicas alternativas, que permitan tanto confirmar como matizar y profundizar el conocimiento del objeto de estudio.

Finalmente, cerramos esta reflexión con una idea de Sumaryanta et al. (2019), quienes afirman que las demandas de una educación de calidad no serán correspondidas si los/as educadores/as no tienen la oportunidad de empoderarse a sí mismos. En términos parecidos, Gómez et al. (2014) también consideran un tema central el empoderamiento en la formación competencial de los/as futuros/as educadores/es. Y es que, de hecho, las competencias de los/as profesionales de la educación se reflejan y reproducen en las expectativas de los/as jóvenes a los que acompañan (Flores, 2015). Las estrategias que ponen en juego, la metodología utilizada y los recursos aplicados condicionan, amplifican e inciden en los resultados educativos y, en este caso, en el empoderamiento juvenil como resultado final.

\section{NOTAS}

1 Proyecto HEBE. Identificación de factores potenciadores y limitadores del empoderamiento juvenil: Análisis de discursos y prácticas de educadores. Programa Estatal de I+D+I Retos de la Sociedad 2017 del MINECO. EDU2017-83249-R. http://www.projectehebe.com/ 


\section{REFERENCIAS BIBLIOGRÁFICAS}

Bauman, Z. (2010). Mundo consumo. Paidós.

Belykh, A. (2018). Resiliencia e inteligencia emocional: conceptos complementarios para empoderar al estudiante. Revista Latinoamericana de Estudios Educativos, 48(1), 255-282.

Collura, J., Raffle, H., Collins, A., \& Kennedy, H. (2019). Creating spaces for young people to collaborate to create community change: Ohio's youth-led initiative. Health Education \& Behavior, 46(1S) 44S-52S. https:// doi.org/10.1177/1090198119853571

Council of Europe. (2015). Council of Europe Youth Work Portfolio. A tool for the assessment and development of youth work competence. Council of Europe.

Davidson, E., \& Carlin, E. (2019). "Steeling" young people: Resilience and youth policy in Scotland. Social Policy and Society, 18(3), 479-489. https://doi. org/10.1017/S1474746419000095

Deutsch, N., Mauer, V., Johnson, H., Grabowska, A., \& Arbeit, M. (2020). "[My counselor] knows stuff about me, but [my natural mentor] actually knows me": Distinguishing characteristics of youth's natural mentoring relationships. Children and Youth Services Review, 111 (104879). https://doi.org/10.1016/j. childyouth.2020.104879

Flores, A. (2015). Empowerment and civic surrogacy: Community workers' perceptions of their own and their latino/a students' civic potential. Anthropology \& Education Quarterly, 46(4), 397-413. https://doi.org/10.1111/ aeq. 12118

Froufe, S. (1997). Los ámbitos de intervención en la Educación Social.
Aula, 9, 179-200. https://revistas. usal.es/index.php/0214-3402/article/ view/3491

Gómez, I. M., Lledó, A., Parandones, T. M., \& Herrera, L. (2014). El empoderamiento como estrategia de éxito en la formación inicial del profesorado. International Journal of Developmental and Educational Psychology, 7(1), 151-160. https://doi. org/10.17060/ijodaep.2014.n1.v7.786

Gonçalves, M., \& García, À. (2016). Education and struggles for recognition: the strategic role of empowerment. Revista Española de Pedagogía, 264, 283-296.

Goyette, M., Mann-Feder, V., Turcotte, D., \& Grenier, S. (2016). Youth empowerment and engagement: an analysis of support practices in the youth protection system in Québec. Revista Española de Pedagogía, 74, 3149.

Greene, S., Burke, K., \& McKenna, M. K. (2018). A review of research connecting digital storytelling, photovoice, and civic engagement. Review of Educational Research, 88(6), 844-878. https://doi. org/10.3102/0034654318794134

Greeson, J., Treglia, D., Morones, S., Hopkins, M., \& Mikell, D. (2020). Youth Matters: Philly (YMP): Development, usability, usefulness, $\&$ accessibility of a mobile web-based app for homeless and unstably housed youth. Children and Youth Services Review, 108(104586). https://doi. org/10.1016/j.childyouth.2019.104586

Hongyee, G. (2020). A comparative analysis of online, offline, and integrated counseling among hidden youth in Hong Kong. Children 
and Youth Services Review, 114, 105042. https://doi.org/10.1016/j. childyouth.2020.105042

Kirk, C. M., Lewis, R. K., Brown, L. K., Karibo, B., Scott, A., \& Park, E. (2015). The Empowering Schools Project: Identifying the Classroom and School Characteristics That Lead to Student Empowerment. Youth \& Society, 49(6), 827-847. https://doi. org/10.1177/0044118X14566118

Paños, J. (2017). Educación emprendedora y metodologías activas para su fomento. Revista Electrónica Interuniversitaria de Formación del Profesorado, 20(3), 33-48. https://doi. org/10.6018/reifop.20.3.272221

Peterson, N. A. (2014). Empowerment theory: clarifying the nature of higherorder multidimensional constructs. American journal of community psychology, 53(1-2), 96-108. https://doi. org/10.1007/s10464-013-9624-0

Prowell, A. N. (2019). Using poststructuralism to rethink risk and resilience: Recommendations for social work education, practice, and research. Social Work, 64(2), 123-130. https://doi.org/10.1093/sw/swz007

Rappaport, J. (1984). Studies in empowerment: Introduction to the Issue. Prevention in Human Services, 38(2-3), 1-7. https://doi.org/10.1300/ J293v03n02_02

Rojas, L. (2014). Unstandardizing teaching: The classroom teacher as an institutional and empowerment agent for Latinalo youth's college access. Doctoral Dissertation, California State University.

Ruff, D., \& Harrison, K. (2020). "Ask Me What I Want": Communitybased participatory research to explore transition-age foster Youth's use of support services. Children and Youth Services Review, 108, 104608. https://doi.org/10.1016/j. childyouth.2019.104608

San Pedro, P. (2006). El Individuo como agente del cambio: El proceso de empoderamiento. FRIDE Desarrollo «En Perspectiva», 1, 1-8.

Soler, P., Trilla, J., Jiménez-Morales, M., \& Úcar, X. (2017). La construcción de un modelo pedagógico del empoderamiento juvenil: espacios, momentos y procesos. Pedagogía Social. Revista Interuniversitaria, 30, 19-32. https://doi.org/10.7179/ PSRI_2017.30.02

Sullivan, A., \& Simonson, G. (2016). A systematic review of school-based social-emotional interventions for refugee and war-traumatized youth. Review of Educational Research, 86(2), 503-530. https://doi. org/10.3102/0034654315609419

Sumaryanta, Mardapi, D., Sugiman \& Herawan, T. (2019). Community-based teacher training: Transformation of sustainable teacher empowerment strategy in Indonesia. Journal of Teacher Education for Sustinability, 21(1), 48-66. https://doi.org/10.2478/ jtes-2019-0004

Sung, Y-T., Yang, J-M., \& Lee, H-Y. (2017). The effects of mobilecomputer-supported collaborative learning: Meta-analysis and critical synthesis. Review of Educational Research, 87(4), 768-805. https://doi. org/10.3102/0034654317704307

To, S.-M., Chun-Sing, J., Liu, X., Danielle, C., Junfei, H., \& Man-yuk, A. (2020). Youth empowerment in the community and young people's creative self-efficacy: The moderating role of youth-adult partnerships in youth service. Youth and Society, 
53(6), 1021-1043. https://doi. A social justice youth development

org/10.1177/0044118X20930890

Úcar, X., Jiménez-Morales, M., Soler,

P., \& Trilla, J. (2016). Exploring the

conceptualization and research of

empowerment in the field of youth.

International Journal of Adolescence

and Youth, 22(4), 405-418. https://doi.

org/10.1080/02673843.2016.1209120

Vázquez-Fernández, M. J. \& Barrera-

Algarín, E. (2016). The essential role

of the social worker as an integration

agent for the youth who face school

failure. The British Journal of Social

Work, 47(7), 1923-1939. https://doi.

org/10.1093/bjsw/bcw137

Wagaman, A. M. (2016). Promoting empowerment among LGBTQ youth: approach. Child and Adolescent Social

Work Journal, 33, 395-405. https://doi.

org/10.1007/s10560-016-0435-7

Young, P., Hong, R., \& Choi, S. (2020).

Validation of the Employment Hope

Scale (EHS) among summer youth

employment program participants.

Children and Youth Services Review,

111, 104811. https://doi.org/10.1016/j.

childyouth.2020.104811

Zimmerman, M. (2000). Empowerment

theory: psychological, organizational

and community levels of analysis. In

J. Rappaport \& E. Seidman (Eds.),

Handbook of Community Psychology

(pp. 43-63). Springer Science \& Business Media. 


\section{PERFIL ACADÉMICO Y PROFESIONAL DE LOS AUTORES}

Carme Trull-Oliva. ORCID: https://orcid.org/0000-0003-2777-0315

Técnica de Investigación Especializada (Área de Estudios de Educación y Psicología) y Profesora Asociada (Área de Teoría e Historia de la Educación) de la Universitat de Girona. Grado en Educación Social, Máster Interuniversitario en Juventud y Sociedad, y Doctorado en Educación. Miembro de Liberi. Grupo de Investigación en Infancia, Juventud y Comunidad del Instituto de Investigación Educativa de la UdG y de la SIPS (Sociedad Iberoamericana de Pedagogía Social). E-mail: carme.trull@udg.edu

Àngela Janer Hidalgo. ORCID: https://orcid.org/0000-0002-4155-2472

Profesora Ayudante Doctora del Área de Métodos de Investigación y Diagnóstico en Educación (MIDE) de la Universidad Internacional 
de la Rioja. Miembro del grupo de investigación GAPEF (Análisis de Políticas Educativas de Formación) del Departamento de Pedagogía Sistemática y Social de la Universitat Autònoma de Barcelona. Coordinadora del grupo JIPS (Jóvenes Investigadores de la Sociedad Iberoamericana de Pedagogía Social). E-mail: angelajaner@gmail.com

Laura Corbella Molina. ORCID: https://orcid.org/0000-0002-2151-089X

Investigadora predoctoral del departamento de Teorías de la Educación y Pedagogía Social de la Universitat Autònoma de Barcelona. Máster en investigación en educación dentro de la especialidad de desigualdades, investigación y acción educativa (UAB) y graduada en Educación social (UdL). Miembro del grupo de investigación GIPE - GAPEF (Grupo Interdisciplinario de Políticas Educativas - Grupo de Análisis de Políticas Educativas y de Formación) y de la SIPS (Sociedad Iberoamericana de Pedagogía Social). E-mail: laura.corbella@uab.cat

Pere Soler-Masó. ORCID: http://orcid.org/0000-0002-8636-0925

Profesor titular de universidad en el Departamento de Pedagogía de la Universitat de Girona. Investigador principal de Liberi. Grupo de Investigación en Infancia, Juventud y Comunidad del Instituto de Investigación Educativa de la UdG. Coordinador desde el 2008 del Máster Oficial Interuniversitario en Juventud y Sociedad (MIJS) impartido por las universidades de Girona, Barcelona, Autónoma de Barcelona, Rovira i Virgili, Lleida y Pompeu Fabra. E-mail: pere. soler@udg.edu

Juan González-Martínez. ORCID: https://orcid.org/0000-0002-9175-6369

Profesor Agregado Serra Húnter del Departamento de Pedagogía de la Universitat de Girona. Doctor en Lingüística Aplicada (UNED) y Tecnología Educativa (URV). Licenciado en Antropología Social y Cultural (UNED). Miembro del grupo de Investigación UdiGital.Edu de la UdG y profesor del Doctorat en Educació de la UdG y del Doctorado en Educación Multimodal de la Universidad Autónoma de Querétaro. Coordinador del Grado de Maestro/a en Educación Primaria. E-mail: juan.gonzalez@udg.edu

Fecha Recepción del Artículo: 16. Febrero. 2021

Fecha Modificación del Artículo: 16. Mayo. 2021

Fecha Aceptación del Artículo: 14. Julio. 2021

Fecha Revisión para Publicación: 13. Septiembre. 2021 
CARME TRULL-OLIVA, ÁNGELA JANER HIDALGO, LAURA CORBELLA MOLINA,

PERE SOLER-MASÓ, JUAN GONZÁLEZ-MARTÍNEZ

SOBRE LAS ESTRATEGIAS METODOLÓGICAS DE LOS/AS EDUCADORES/AS PARA CONTRIBUIR... 